\title{
The 5th Summit on Chinese Psychiatric Genetics: Multi-Omics and Spectrum of Psychiatric Disorders
}

Chunyu Liu

Department of Psychiatry, SUNY Upstate Medical University, Syracuse, NY 13201, USA; Email: liuch@upstate.edu.

\section{INTRODUCTION}

To promote research in psychiatric genetics in China, Global Diversity Task Force of the International Society of Psychiatric Genetics headed by Chunyu Liu, together with other ISPG members, organized annual "Summit on Chinese Psychiatric Genetics" since 2004. This year (2018), the 5th Summit on Chinese Psychiatric Genetics with the theme "Multi-Omics and Spectrum of Disorders" was held in the Affiliated Guangji Hospital of Soochow University in Suzhou, China on May 20th. It also served as the first meeting of the Genetics working group since the establishment of the Mental Health Branch of China International Exchange and Promotive Association for Medical and Health Care. In the two-day meeting, ten senior investigators shared their latest research results. Fourteen trainees and junior investigators also presented their works to compete for the JPBS Travel Awards. It covered the latest achievements and topics of current interest in the study of psychiatric genetics in China.

\section{SELECTED INVITED LECTURES BY SENIOR INVESTIGATORS}

Steve Faraone from the SUNY Upstate Medical University presented an update review of genetic studies of ADHD (Attention Deficit Hyperactivity Disorder). He not only reviewed the findings of common SNP (Single Nucleotide Polymorphisms) and rare variants, but also presented the latest results of pharmacogenetics, and discussed the potential use of genetics for ADHD diagnosis.

Jianping Zhang from the Zucker Hillside Hospital presented a genetic study of antipsychotic drug efficacy in four cohorts of first episode schizophrenia patients with a total of more than five 
hundred subjects. He provided strong evidence that the treatment response is inversely correlated with schizophrenia polygenic risk score. Higher risk score is associated with higher symptoms at 12 weeks, less symptom reduction or poorer antipsychotic efficacy.

Another important pharmacogenetics study was presented by Thomas G. Schulze from Institute of Psychiatric Phenomics and Genomics (IPPG), Ludwig-Maximilans-University of Munich (LUM). Thomas G. Schulze has led the International Consortium of Lithium Genetics (ConLiGen) since 2008, which has published several impactful papers showing genetics associated with lithium treatment response in bipolar disorder patients. Their latest study showed that the core genes of circadian rhythms may involve the dichotomous lithium response in patients. ConLiGen invites participation from more investigators in China to expand their subject size to over 5,000 .

Hong Xue from the Hong Kong University of Science and Technology shared their mouse Gabrb2 (gamma-aminobutyric acid type A receptor beta 2 subunit) knockout model study, which showed interneuron-astrocyte-microglia dysregulation and schizophrenia-like behavior.

Chao Chen from the Central South University presented their new method, csuWGCNA (combining the signed and unsigned Weighted Gene Co-expression Network Analysis), for coexpression network analysis. His csuWGCNA was modified with WGCNA by targeting both positive and negative correlations among coexpressed genes. Such modification made it more sensitive in capturing negative regulations that could be missed by classic WGCNA method.

Hailiang Huang from the Stanley Center for Psychiatric Research at Broad Institute presented their schizophrenia genetic study cross ancestry groups. He aggregated samples of the Asian ancestry and compared the schizophrenia genetic architecture in Asian and European ancestries. The study found that the two ancestries have the same SNP heritability, almost perfect genetic correlation, and similar pathways implicated. Despite that, he showed that Asian and European ancestries have different allele frequencies for many schizophrenia SNPs, leading to findings unique to one specific population. He used population diversity to improve mapping resolution and predicted causal variants. The transferability of polygenic risk score across populations was also empirically assessed.

Zhongming Zhao from the University of Texas,
Health Science Center at Houston shared his new bioinformatics study of schizophrenia genetics. They used Mega-analysis of Odds Ratio (MegaOR) to gain deep understanding of genetic findings in schizophrenia.

Jubao Duan from the University of Chicago presented their study on iPSC (induced pluripotent stem cell) models of mental disorders. He used ATAC (Assay for Transposase-Accessible Chromatin using sequencing)-seq and RNA-seq data of three time-points from iPSC-derived neuron to study the dynamics of chromatin states and gene expression in the process of neuronal differentiation. He identified gene expression networks regulated by transcription factors NGN2 (Neurogenin 2) and NEUROD1 (Neuronal Differentiation 1). He also developed methods to study Allele-specific open chromatin (ASoC) as a functional readout of regulatory variants, and showed that ASoC was prevalent in iPSCderived neurons. He further used CRISPR/dCas9 to edit genome and showed that chromatin states change gene expression in neuronal progenitor cells.

Chunyu Liu from the SUNY Upstate Medical University presented updates of the PsychENCODE project, which is the biggest epigenomic study of psychiatric brain. His talk reviewed the brief history of PsychENCODE, the major data and results produced by this project. It is expected that PsychENCODE will bring a breakthrough in understanding genomics and epigenomics of psychiatric brains.

Liyuan Guo from the Institute of Psychology, of CAS Key Laboratory of Mental Health introduced their web-based databases rSNPbases and rVarBase. These databases are valuable tools for annotating SNP by integrating data from many sources to predict their regulatory functionality and associated networks. They could be important resource for investigators in the field of psychiatric genetics.

\section{SELECTED ABSTRACTS}

There were fourteen trainees or junior investigators running for best oral presentations competition and the followings were selected as the best oral presentations. Each of three winnersRujia Dai, Chen Liang and Fuquan Zhang, was awarded 1,000 USD travel grant supported by JPBS. They will attend the ISPG annual meeting (XXVI World Congress of Psychiatric Genetics) in Glasgow, Scotland. 
(1) Cell Type-Specific Alternation in Schizophrenia and Bipolar Disorder

Rujia Dai ${ }^{1}$, Lulu Chen ${ }^{2}$, Yu Chen ${ }^{1}$, Sihan Liu ${ }^{1}$, Jiacheng Dai ${ }^{1}$, Guoqiang $\mathrm{Yu}^{2}$, Yue Wang ${ }^{2}$, Chao Chen ${ }^{1,3}$, Chunyu Liu ${ }^{1,4}$

1 School of Life Sciences, Central South University, Changsha 410083, China

2 Department of Electrical and Computer Engineering, Virginia Polytechnic Institute and State University, VA 24061, USA

3 National Clinical Research Center for Geriatric Disorders, Xiangya Hospital, Central South University, Changsha 410083, China

4 Department of Psychiatry, SUNY Upstate Medical University, Syracuse, NY 13201, USA

Background: The brain consists of hundreds of cell types. The cell type diversity is the basis of the complex brain functions, also neuropsychiatric diseases. Many works discovered that the psychiatric disorders are associated with specific cell types. But how these cell types are involved in psychiatric pathogenesis is largely uncertain.

Methods: We used a comprehensive transcriptomic resource of 413 dorsal lateral prefrontal cortex samples from PsychENCODE project with 2 major psychiatric disorders, SCZ and $\mathrm{BD}$, and controls, and applied a novel method called "sample-specific CAM (csCAM)" to fully deconvolute the RNA-Seq data of brain bulk tissue data into astrocyte-specific, neuronspecific, microglia-specific and oligodendrocytespecific expression metrics. Further, we estimated cell proportion, analyzed cell type-specific differential gene expression (DGE) and co-expression pattern (COE) associated with disease, and identified cell type-specific eQTL (cs-eQTL). Using MAGMA and TWAS, we further tested published GWAS signals enrichment in the co-expression modules and cs-eQTL.

Results: We found the proportions of microglia and oligodendrocytes are increasing in the disease (SCZ + BD) groups (Wilcoxon test, $\left.p_{\text {micro }}=1.05 \times 10^{-6}, p_{\text {oligo }}=0.006\right)$, but neuron is decreasing (Wilcoxon test, $p_{\text {neuron }}=4.26 \times 10^{-6}$ ). In total, 2831 genes showed cell-type-specific differential expression (FDR $<0.05)$. The numbers of cell type-specific DGE (cs-DGE) genes is 303 for astrocyte, 8 for microglia, 617 for the neuron, and 453 for oligodendrocyte. Remarkably, 2/3 of the CS-DGE genes cannot be detected in bulk data. Setting a 2-fold cutoff, the number of downregulated genes respectively is $7,3,108,11$ and the number of upregulated genes is $7,24,45,4$ for astrocyte, microglia, neuron and oligodendrocyte. The number of upregulated genes is $7,24,45,4$ for astrocyte, microglia, neuron and oligodendrocyte. By constructing cell type-specific co-expression network, we identified disease-related modules in a cell type-specific manner. Using MAGMA, we observed significant enrichment of aggregate GWAS signal among the disease-related modules in all cell types. The most significant disease-related module is related to negative regulation of kinase activity $\left(p=1.56 \times 10^{-8}\right)$ and negative regulation of phosphorylation $\left(p=9.57 \times 10^{-8}\right)$ in the neuron, mitochondrial translation $\left(p=4.48 \times 10^{-4}\right)$ in astrocyte, activation of immune response $\left(p<10^{-8}\right)$ in microglia and vesicle docking $\left(p=4.29 \times 10^{-4}\right)$ in oligodendrocyte. We found that the co-expression of immune-related genes are shared in all cell types, and the correlation of corresponded module eigengene is 0.65-0.82. We identified more than 12 million eQTL (FDR $<0.05)$ by combining genotype data and cell type-specific expression. In these eQTLs, only $\sim 5 \%$ $(n=585,944)$ of the eQTL is shared by four cell types and $\sim 62 \%(7,403,586)$ of them are cell type-specific (cs-eQTL). The neuron has the most amount of eQTLs $(n=2,776,332)$, and oligodendrocyte has the most eQTLs in the glial cells $(n=1,761,374)$. We downloaded the 108 genetic loci associated with schizophrenia and 30 genetic loci associated with bipolar disorder from Psychiatric Genomics Consortium. In total, 17 bipolar loci and 68 schizophrenia loci are cs-eQTL SNP. Seventeen of 68 schizophrenia loci are only detected by one cell type $\left(n_{\text {neuron }}=7, n_{\text {microglia }}=4, n_{\text {oligodendrocyte }}=6\right)$, such as rs35518360, rs12826178, rs7267348. Three of 17 bipolar loci are only detected by one cell type $\left(n_{\text {neuron }}=1, n_{\text {microglia }}=1, n_{\text {oligodendrocyte }}=1\right)$, which is rs113779084, rs11647445 and rs9834970.

Conclusions: We showed that the cell type has both specific and common changes in the process of the psychiatric disorder, especially in the aspect of cell composition, gene expression and gene regulation. More importantly, some disease-associated variants operate in a cell-type-specific manner. These findings provide new insights into cell type-specific molecular alterations in SCZ and BD.

\section{(2) Exploring the Neurodevelopmental Trajectory and Modifier gene of 16p11.2 Microdeletion Using intra and Inter-Family Carriers}

Hua Xie ${ }^{1}$, Fang Liu ${ }^{1}$, Nan $\mathrm{Wu}^{2}$, Yu Zhang ${ }^{1}$, Yiping Shen ${ }^{3,4,5}$, Jame F Gusella ${ }^{5}$, Jian Wang ${ }^{3}$, Xiaoli Chen ${ }^{*}$ 
1 Department of Medical Genetics, Capital Institute of Pediatrics, Beijing 100000, China

2 Department of Orthopedic Surgery, Peking Union Medical College Hospital, Peking Union Medical College and Chinese Academy of Medical Sciences, Beijing 100000, China

3 Shanghai Children's Medical Center, School of Medicine, Shanghai Jiaotong University, Shanghai 200000, China

4 Department of Pathology, Harvard Medical School, Boston, MA 02115, USA

5 Molecular Neurogenetics Unit, Center for Genomic Medicine, Massachusetts General Hospital, Boston, MA, 02114, USA

Background: Neurodevelopmental disorders (NDDs) are a group of pediatric disorders with severely affected behavioral features caused by alterations in early brain development. Causative genetic variants in NDDs can range in size from the substitution, deletion or duplication of a single base pair, to structural variants and to altered copy numbers of an entire chromosome (aneuploidy) or genome. The $600 \mathrm{~kb}$ deletion region at 16p11.2 between 29.5 and $30.1 \mathrm{Mb}$ is a typical recurrent copy number variation (CNV), which has been implicated as an important genetic risk of NDDs. $16 \mathrm{p} 11.2$ deletion carriers present heterogeneity of phenotype, from completely healthy to different degrees of autism spectrum disorders (ASDs) or intellectual disability (ID), and its potential pathogenesis is unknown.

Methods: 212 Chinese children with unknown NDD were recruited from the affiliated Children's Hospital of Capital Institute of Pediatrics. The FMR1 CGG triplet-repeat primed (TP)-PCR assay was used to rule out FMR1 full mutation. Array comparative genomic hybridization (aCGH) was used to identify 16 p11.2 deletion in NDD children. Targeted sequencing for the 25 coding genes in $16 p 11.2$ interval was performed in $16 \mathrm{p} 11.2$ deletion carriers, exploring the potential modifier gene involved in NDD phenotypic heterogeneity.

Results: Two independent children with 16p11.2 deletion were identified, giving that the prevalence of $16 \mathrm{p} 11.2$ deletion in Chinese NDD cohort is $0.94 \%$ (2/212). Both two 16p11.2 deletions are paternal inherited. The result of targeted sequencing in seven $16 \mathrm{p} 11.2$ deletion carriers showed only one SNP (rs10204) on 3'-UTR of PRRT2 gene releases consistently segregation in six NDD patients (" $\mathrm{C}$ " genotype) and one healthy carrier ("T" genotype).

Conclusions: This study gave the prevalence of 16p11.2 deletion in Chinese children with NDD, which hinted that $16 p 11.2$ deletion is the same genomic pathogenicity of NDD as other known ones. The 16p11.2 deletion combined a SNP (rs10204) of PRRT2 on the hemizygous allele may be the modifier gene of NDD phenotypic penetrance of $16 \mathrm{p} 11.2$ deletion.

(3) Common Variants of the LINC00461 Gene Affect Expression, Neuronal Migration, Hippocampus Structure, Cognitive Performance and Schizophrenia Susceptibility

Shuquan Rao', Jun $\mathrm{Li}^{2,3}$, Yong $\mathrm{Xu}^{4}$, Bernard Lerer $^{5}$, Ana Alkelai ${ }^{5}$, Sudha Seshadri ${ }^{6,7}$, Susanne $\mathrm{Erk}^{8}$, Henrik Walter ${ }^{8}$, Heike Tost ${ }^{9}$, Andreas Meyer-Lindenberg ${ }^{9}$, Zhiqiang $\mathrm{Li}^{10}$, Lei Cai ${ }^{10}$, Marcella Rietschel ${ }^{11}$, Franziska Degenhardt ${ }^{12,13}$, Markus M. Nöthen ${ }^{14,15}$, Sven Cichon ${ }^{14-18}$, the MooDSConsortium ${ }^{19}$, Yongyong Shi ${ }^{10}$, Lin $\operatorname{Tian}^{20}$, Chunhui $\mathrm{Jin}^{20}$, Weihua Yue ${ }^{2,3}$, Fuquan Zhang ${ }^{20}$

1 School of Life Science and Engineering, Southwest Jiaotong University, Chengdu 610031, China

2 Department of Biochemistry, Institute of Mental Health, the Sixth Hospital, Peking University, Beijing 100871, China

3 Key Laboratory of Mental Health, Ministry of Health \& National Clinical Research Center for Mental Disorders, Peking University, Beijing 100871, China

4 Department of Psychiatry, First Clinical Medical College/First Hospital of Shanxi Medical University, Taiyuan 030001, China

5 Biological Psychiatry Laboratory, Department of Psychiatry, Hadassah-Hebrew University Medical Center, Jerusalem 91120, Israel

6 Department of Neurology, Boston University School of Medicine, Boston, MA 02118, USA

7 Glenn Biggs Institute for Alzheimer's and Neurodegenerative Diseases, University of Texas Health Sciences Center, San Antonio, TX 78229, USA

8 Department of Psychiatry and Psychotherapy, Charité Universitätsmedizin, Berlin 10117, Germany

9 Department of Psychiatry and Psychotherapy, Central Institute of Mental Health, Medical Faculty Mannheim, University of Heidelberg, Mannheim 69117, Germany 
10 Key Laboratory for the Genetics of Developmental and Neuropsychiatric Disorders, Bio-X Institutes, Shanghai Jiao Tong University, Shanghai 200030, China

11 Department of Genetic Epidemiology in Psychiatry, Central Institute of Mental Health, University of Heidelberg, Mannheim, Germany

12 Institute of Human Genetics, University of Bonn School of Medicine \& University Hospital Bonn, Bonn, Germany

13 Department of Genomics, Life \& Brain Center, School of Medicine, University of Bonn, Bonn 53127, Germany

14 Institute of Medical Genetics and Pathology, University Hospital Basel, Basel 4031, Switzerland

15 Department of Biomedicine, University of Basel, Basel 4031, Switzerland

16 Department of Genomics, Life and Brain Center, University of Bonn, Bonn 53127, Germany

17 Institute of Human Genetics, University of Bonn, Bonn 53115, Germany

18 Institute of Neuroscience and Medicine (INM-1), Research Center Juelich, Juelich, Germany

19 Members of the Moods Consortium: Thomas W. Mühleisen, Markus Leber, Franziska Degenhardt, Jens Treutlein, Manuel Mattheisen, Anna Maaser, Sandra Meier, Stefan Herms, Per Hoffmann, André Lacour, Stephanie H. Witt, Fabian Streit, Susanne Lucae, Wolfgang Maier, Markus Schwarz, Helmut Vedder, Jutta Kammerer-Ciernioch, Andrea Pfennig, Michael Bauer, Martin Hautzinger, Adam Wright, Janice M. Fullerton, Grant W. Montgomery, Sarah E. Medl and, Scott D. Gordon, Tim Becker, Johannes Schumacher, Peter Propping

20

Department of Psychiatry, Wuxi Mental Health Center of Nanjing Medical University, 156, Qianrong Road, Wuxi, 214151, China

Schizophrenia is a neurodevelopmental disorder that has shown evidence of genetic overlap and substantial evidence of pleiotropy with brain structure and cognitive function, but the genes and molecular mechanisms accounting for this have been challenging to identify. Here, genetic evidence was presented from both Caucasian and Chinese populations $(\sim 120,000$ subjects) revealing the long intergenic non-protein coding RNA 461 (LINC00461) as a novel susceptibility gene for schizophrenia. Single-nucleotide polymorphisms (SNPs) located in regulatory elements spanning the LINC00461 region are significantly associated with schizophrenia (index SNP rs410216, $\left.p_{\text {meta }}=1.43 \times 10^{-5}, \mathrm{OR}=1.050\right)$; subjects carrying the risk allele of rs410216 showed decreased hippocampus volume $\left(p=1.43 \times 10^{-5}, \beta=-12.10\right)$, impaired cognitive performance $\left(p=6.03 \times 10^{-5}\right.$, $\beta=-0.011)$ and increased anxiety symptoms $(p=0.032$, OR $=1.062)$, as compared with non-carriers. Moreover, the risk allele predicts lower transcriptional level of LINC00461 in the hippocampus. Furthermore, reduced expression of LINC00461 in the mice hippocampus elicits behavioral changes, including the prepulse inhibition (PPI), conditional contextual memory or open field, with translational relevance to cognitive function. Finally, delayed neuronal migration from the intermediate zone (IZ) to cortical plate (CP) during the cortical development is induced by the knockdown of LINC00461 expression. Together, these convergent lines of evidence implicate inadequate LINC00461 expression in the hippocampus in the development of schizophrenia, providing novel insight into the genetic architecture and biological etiology of schizophrenia.

\section{CONCLUSIONS}

The 5th Summit on Chinese Psychiatric Genetics was a success in its aims of increasing public awareness of the importance of genetic research in China and promoting participation of clinicians in psychiatry genetic testing.

This Summit hosted 10 invited talks and 14 JPBS travel award competition presentations within two days. This report presents an overview of novel research findings emerged during 5th Summit on Chinese Psychiatric Genetics. We hope those highlights in this report could encourage interested researchers to join us at our next meeting in Shenzhen. 